\title{
General Psychiatry Effects of regulating intestinal microbiota on anxiety symptoms: A systematic review
}

Beibei Yang, Jinbao Wei, Peijun Ju, Jinghong Chen

To cite: Yang B, Wei J, Ju P, et al. Effects of regulating intestinal microbiota on anxiety symptoms: A systematic review. General Psychiatry 2019;32:e100056. doi:10.1136/ gpsych-2019-100056

Received 23 January 2019 Revised 28 February 2019 Accepted 03 March 2019

Check for updates

(C) Author(s) (or their employer(s)) 2019. Re-use permitted under CC BY-NC. No commercial re-use. See rights and permissions. Published by BMJ.

Shanghai Key Laboratory of Psychotic Disorders, Shanghai Mental Health Center, Shanghai Jiao Tong University School of Medicine, Shanghai, China

Correspondence to Dr Jinghong Chen; chenjh_008@hotmail.com

\section{ABSTRACT}

Background Anxiety symptoms are common in mental diseases and a variety of physical disorders, especially in disorders related to stress. More and more basic studies have indicated that gut microbiota can regulate brain function through the gut-brain axis, and dysbiosis of intestinal microbiota was related to anxiety. However, there is no specific evidence to support treatment of anxiety by regulating intestinal microbiota.

Aims To find evidence supporting improvement of anxiety symptoms by regulation of intestinal microbiota.

Methods This systematic review of randomised controlled trials was searched based on the following databases: PubMed, EMBASE, the Cochrane Library, OVID, Web of Knowledge, China National Knowledge Infrastructure (CNKI), Wanfang Data, VIP databases and SinoMed. The retrieval time dated back to 25 July 2018. Then we screened research literatures based on established inclusion and exclusion criteria. Quality evaluation for each included study was done using the Cochrane risk of bias and the Jadad scale.

Results A total of 3334 articles were retrieved and 21 studies were included which contained 1503 subjects. In the 21 studies, 14 chose probiotics as interventions to regulate intestinal microbiota and six chose non-probiotic ways such as adjusting daily diets. Probiotic supplements in seven studies contained only one kind of probiotic, two studies used a product that contained two kinds of probiotics and the supplements used in the other five studies included at least three kinds of probiotics. In the studies that used treatment as usual plus interventions regulating intestinal flora (IRIF) as interventions (five studies), only non-probiotic ways were effective (two studies), which means $40 \%$ of studies were effective; in the studies that used IRIF alone (16 studies, 11 studies used probiotic ways and 5 studies used non-probiotic ways), $56 \%$ of studies could improve anxiety symptoms, and $80 \%$ of studies that conducted the non-probiotic interventions were effective, while $45 \%$ of studies that used probiotic supplementations had positive effects on anxiety symptoms. Overall, 11 studies showed a positive effect on anxiety symptoms by regulating intestinal microbiota, which indicated $52 \%$ of the 21 studies were effective, and there were five studies that used probiotic supplements as interventions and six used non-probiotic interventions. In addition, it should be noted that six of seven studies showed that regulation of intestinal microbiota could treat anxiety symptoms, the rate of efficacy was $86 \%$.
Conclusions We find that more than half of the studies included showed it was positive to treat anxiety symptoms by regulation of intestinal microbiota. There are two kinds of interventions (probiotic and non-probiotic interventions) to regulate intestinal microbiota, and it should be highlighted that the non-probiotic interventions were more effective than the probiotic interventions. More studies are needed to clarify this conclusion since we still cannot run meta-analysis so far.

\section{BACKGROUND}

Anxiety disorder is a mental disorder with anxiety symptoms as the main clinical manifestation, with a global incidence of $3 \%-25 \%$, and the incidence in chronic diseases, such as cancer, cardiocerebrovascular disease, irritable bowel syndrome (IBS), is $1.4 \%-70 \%$. $^{1}$ Studies $^{2}$ have shown that up to $33.7 \%$ of people will be affected by anxiety symptoms during their lifetime. Those with a longer course of disease are often accompanied by social cognitive impairment, which has serious impact on patients and society. Therefore, the treatment of anxiety is very important. Clinical principles for the treatment of physical diseases with anxiety symptoms are usually based on the relief of somatic symptoms, and the use of psychiatric drugs, psychotherapy and other treatments can be combined under the premise of ensuring treatment efficacy. In China, anxiety symptoms often are confused with somatic symptoms and neglected in clinical practice. ${ }^{3}$ Therefore, the anxiety symptoms often could not be treated timely and effectively.

The trillions of microorganisms located in the gut are called gut microbiota, and they perform important functions in the immune system and metabolism by providing essential inflammatory mediators, nutrients and vitamins. ${ }^{4}$ Besides, Toll-like receptors (TLR) can specifically recognise lipopolysaccharide (LPS) molecules in pathogenic microorganisms, especially TLR4. After the LPS of the gut microbiota activates the TLR, the NF- $\kappa B$ pathway which regulates the expressions 
of many inflammatory mediators and cytokines is activated. The long-term existence of this immune activation can make brain functions change which finally lead to the kinds of mental disorders like anxiety disorder. ${ }^{5-7}$ Furthermore, studies indicated that gut microbiota could have an impact on the function of the hypothalamus-pituitary-adrenal axis which could lead to changes in brain functions. ${ }^{8}$ Additionally, a growing number of basic and clinical studies have shown that intestinal flora can modulate communication between the gut and the brain ${ }^{9}$ via the gut-brain axis, which ${ }^{10}$ mainly includes the nervous system, immune system and endocrine system. When intestinal flora is affected, a series of changes in physical and/or mental symptoms can occur. ${ }^{11}$

Animal studies have demonstrated that germ-free mice pretended to have anxiety-related behaviours and this condition could be changed by regulating gut microbiota. ${ }^{12-16}$ However, there is no consensus on whether anxiety symptoms can be improved by regulating gut microbiota. Therefore, this systematic review was conducted to provide clarification and new ideas for clinical treatment.

\section{METHODS}

\section{Search strategy}

The following databases were searched up to 25 July 2018: PubMed, EMBASE, the Cochrane Library, OVID, Web of Knowledge, China National Knowledge Infrastructure (CNKI), Wanfang Data, VIP Databases and SinoMed. The search terms were as follows: (anxiety OR anxiety disorder OR generalized anxiety disorder OR GAD OR social anxiety disorder OR SAD) AND (intestinal microbiota OR gut bacteria OR enteric microbiome OR gut microbiota OR fecal microbiota OR intestinal flora OR gut flora). The Chinese search terms were “焦虑” or “焦 虑症状”, AND “肠道菌群”. The retrieval strategy and keywords were modified for different databases. At the same time, we conducted literature traceability to find further relevant research.

\section{Inclusion and exclusion criteria of literature \\ Inclusion criteria}

The inclusion criteria were as follows: (1) patients: the subjects were patients with anxiety symptoms no matter what the diagnoses were and all patients should have been assessed by at least one kind of anxiety scale; (2) interventions versus comparisons: (A) treatment as usual (TAU) plus interventions regulating intestinal flora (IRIF, such as the supplementary of probiotic, changing diet habits, and so on) versus TAU, (B) IRIF alone versus placebo; (3) outcomes: the main outcome of the study was the anxiety symptom measured by kinds of anxiety assessment scales, such as the Hospital Anxiety and Depression Scale (HADS), the Beck Anxiety Inventory (BAI), the StateTrait Anxiety Inventory (STAI), and so on; (4) studies: the study must be a clinical randomised controlled study. $\mathbf{3 3 3 4}$ articles were selected after primary search. Databases were as follows: PubMed, Embase, the Cochrane Library, OVID, Web of Knowledge and Chinese National Knowledge Infrastructure (CNKI), Wanfang Data, VIP Databases, SinoMed. The English keywords used for search were anxiety, anxiety disorder, generalized anxiety disorder, GAD, social anxiety disorder, $\mathrm{SAD}$, intestinal microbiota, gut bacteria, enteric microbiome, gut microbiota, fecal microbiota, intestinal flora, gut flora; The Chinese keywords were '焦虑', ‘焦虑症状', ‘肠道菌群”.

1919 duplicate reports were excluded.

The title and abstract of the 1415 articles were screened.

1348 articles were excluded:

-697 reviews;

- 281 animal studies;

-176 studies not matched with the article types;

- 194 non-randomised human clinical intervention studies

The full text of 67 studies were examined for the next selection.

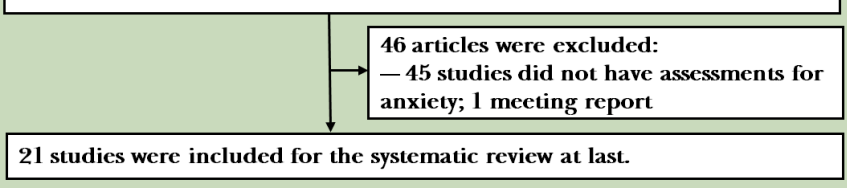

Figure 1 Flowchart of the study. 21 studies were selected for the systematic review after retrieving the databases based on the search strategy.

\section{Exclusion criteria}

The exclusion criteria were: (1) non-clinical randomised controlled trials; (2) non-human studies; (3) reviews; (4) study protocols; (5) data incomplete experiments like meeting reports and so on; and (6) repeated reports.

\section{Literature screening and data extraction}

The literature screening process of this study is shown in figure 1 . The literature search was performed independently by two researchers (B-BY, J-BW) according to the search strategy. If the two search results were different, the two researchers reviewed the literature together and analysed the reasons for the differences. If the opinions were still inconsistent, a third person (P-JJ) would examine and make the final decision. If there was a lack of information in the literature, it was supplemented by contacting the author. We developed the data extraction table for data extraction and verification, and extraction contents included (A) basic information for research; (B) methods of included research; (C) subjects; and (D) interventions and outcomes.

\section{Quality evaluation of literature}

Risk of bias evaluation: the included studies were assessed by two independent investigators based on the risk of bias assessment method recommended by the Cochrane manual version 5.3.0. The specific contents included: (A) random sequence generation; (B) allocation concealment; $(\mathrm{C})$ whether to use the blind method (blinding of the subjects and the treatment providers, blinding of the result evaluators); (D) incomplete results data; and (E) other potential risks affecting authenticity. When there 
were differences between the two evaluators, a third person would make the decision.

Evidence quality assessment: The scoring standards of Jadad scale were as follows: (A) randomisation: (1) the method of randomisation was described and it was appropriate (two points), (2) the study was described as randomised (one point), (3) not randomised or inappropriate method of randomisation (zero point); (B) concealment of allocation: (1) the method of allocation concealment was described appropriately (two points), (2) the study was described as using allocation concealment method (one point), (3) did not describe the method of allocation concealment (zero point); (C) double blinding: (1) the method of double blinding was described and it was appropriate (two points), (2) the study was described as double blind (one point), (3) no blind or inappropriate method of blinding (zero point); (D) withdrawals and dropouts: (1) a description of withdrawals and dropouts (one point), (2) did not describe the follow-up (zero point). One to three points is considered low quality and four to seven points as high quality.

\section{RESULTS}

Basic characteristics of the included literature

The research process is shown in figure 1. A total of 3334 studies were included after retrieving articles from five English databases and four Chinese databases based on the search strategy, and the released deadline of the studies was 25 July 2018. First, 1919 unrelated studies were removed, with 1415 studies remaining in the secondary step. After reading the titles and summaries, 1348 articles were excluded. Finally, after reading the remaining 67 articles, 46 were removed (45 lacking assessments of anxiety and 1 was a meeting report) with 21 studies remaining for the systematic evaluation.

The details of the 21 papers included are shown in table 1. A total of 1503 subjects were included in the 21 studies, including patients with IBS (10 studies), healthy controls (six studies) and other patients with chronic diseases such as: chronic fatigue syndrome (CFS), rheumatoid arthritis (RA), obesity, fibromyalgia and type 2 diabetes mellitus. Five studies conducted TAU plus IRIF when the TAU did not affect the results: three studies used a single kind of probiotic as interventions and two studies conducted non-probiotic ways (supplementary of the resistant dextrin or a diet low in fermentable oligosaccharides, disaccharides, and monosaccharides and polyols [low FODMAP]). The studies that used IRIF alone (16 studies) could be divided into two categories: (1) probiotic interventions (11 studies): (A) single probiotic interventions (four studies), and most of the probiotics were Lactobacillus, (B) two studies used two probiotic mixtures: the Swiss Lactobacillus and the long Bifidobacterium mixture, (C) five studies used at least three probiotic mixtures: Lactobacillus, Streptococcus, Bifidobacterium, and so on; (2) five studies conducted non-probiotic interventions, including low FODMAP, short-chain fructooligosaccharides (scFOS), regulating diet, using trans-galactooligosaccharide mixture, and so on. The most used questionnaires for assessing anxiety symptoms included the HADS (nine studies), the STAI (seven studies), the BAI (two studies), Hamilton Anxiety Rating Scale (HAM-A; two studies), and so on, with five studies choosing two different scales: Sawada and colleagues ${ }^{17}$ and Pinto-Sanchez and colleagues ${ }^{18}$ used HAD and STAI; Messaoudi and colleagues ${ }^{19}$ used HAD and the Hopkins Symptom Checklist-90; Kelly and colleagues ${ }^{20}$ used STAI and BAI; Farhangi and colleagues ${ }^{21}$ used HAM-A and a 42-item self-report questionnaire designed to assess the current severity of symptoms relating to depression, anxiety and stress (Depression, Anxiety and Stress Scale42). Except for Schumann and colleagues ${ }^{22}$ choosing yoga as the intervention in control group, the interventions of the other studies in the control group were matched with the experimental group. All the supplements could not be distinguished by appearance and taste which ensured the blindness of the subjects.

\section{Research quality}

The results of the quality assessment are shown in table 2 . Of all the 21 studies, only Sanchez and colleagues ${ }^{23}$ did not mention methods of random sequence generation, resulting in a rating of 'unclear', and the other studies were all rated as 'low'. According to the Jadad scale, $81 \%$ of the included studies were $\geq 4$ points and assessed as high quality. In addition, 33\% of the studies conducted intentto-treat analysis in order to maintain the random information, ensuring the equilibrium between the groups. Seventeen studies mentioned the rate of withdrawal and/ or dropout, which were $\leq 20 \%$ (the reasons are shown in table 3 ). In summary, the overall quality of the 21 articles included in this study was high.

\section{Therapeutic effects}

Eleven of 21 studies showed that regulation of intestinal microbiota could improve anxiety symptoms, of which five studies conducted probiotic interventions and six studies used non-probiotic interventions like low FODMAP. That means that $52 \%$ of studies showed a positive effect on improving anxiety symptoms by regulating intestinal microbiota, seen in figure 2 . In the five studies that used TAU plus IRIF, the anxiety symptoms were improved all by non-probiotic ways (two studies) while the other three studies that used one kind of probiotic were all invalid. So, we could find that $40 \%$ of studies that used TAU plus IRIF were positive and $56 \%$ of studies that used IRIF alone could improve anxiety symptoms. Anxiety assessment questionnaires included HADS, STAI, BAI and HAM-A. This also indicated that no matter what kinds of measures were taken, and regardless of the assessment scales, anxiety symptoms could be improved by regulating gut microbiota. It is worth mentioning that the efficiency of supplementation of non-probiotic preparations is as high as $86 \%$, which suggested that in addition to supplementing probiotics, it is worth noticing by clinicians to 


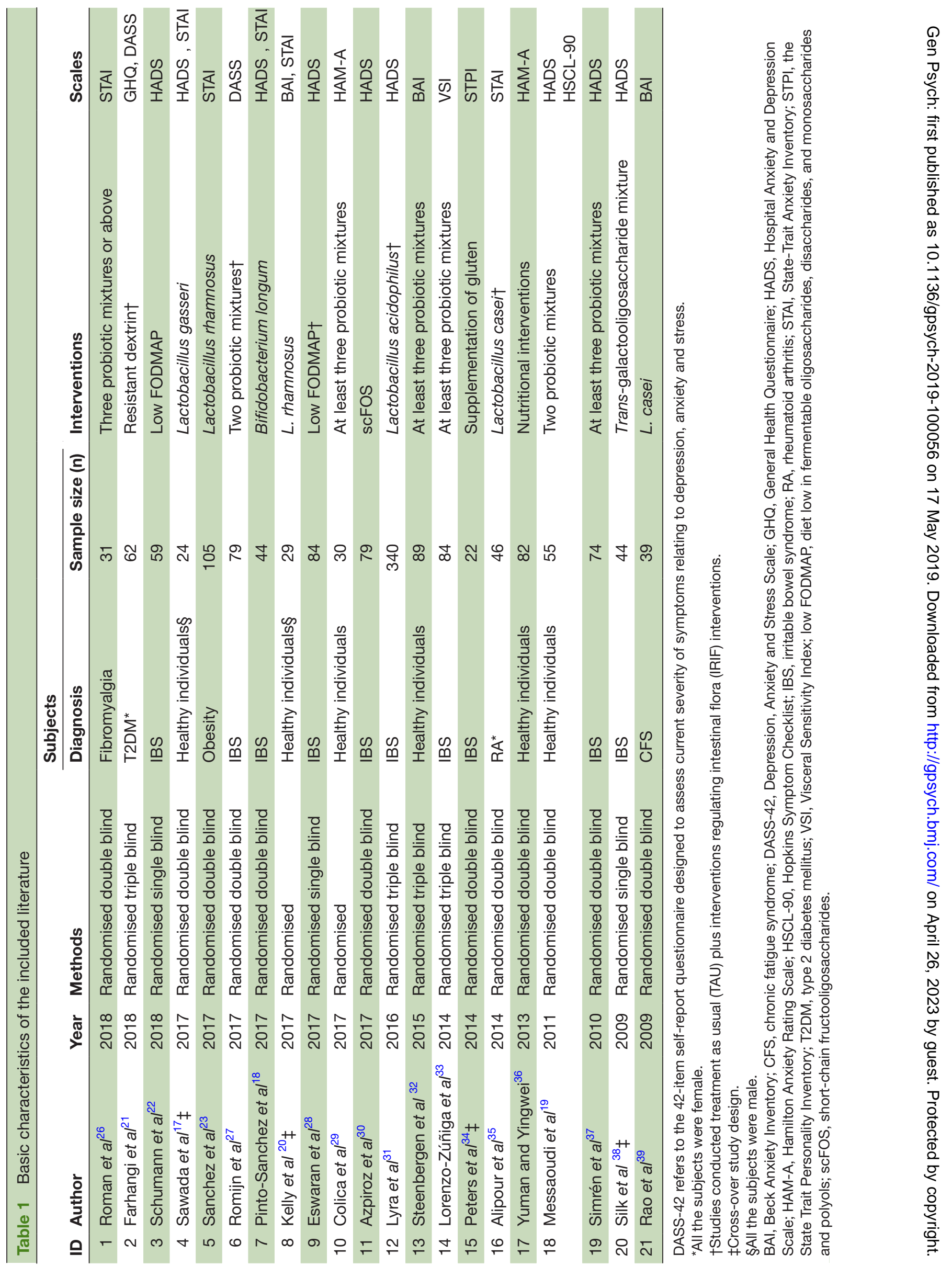




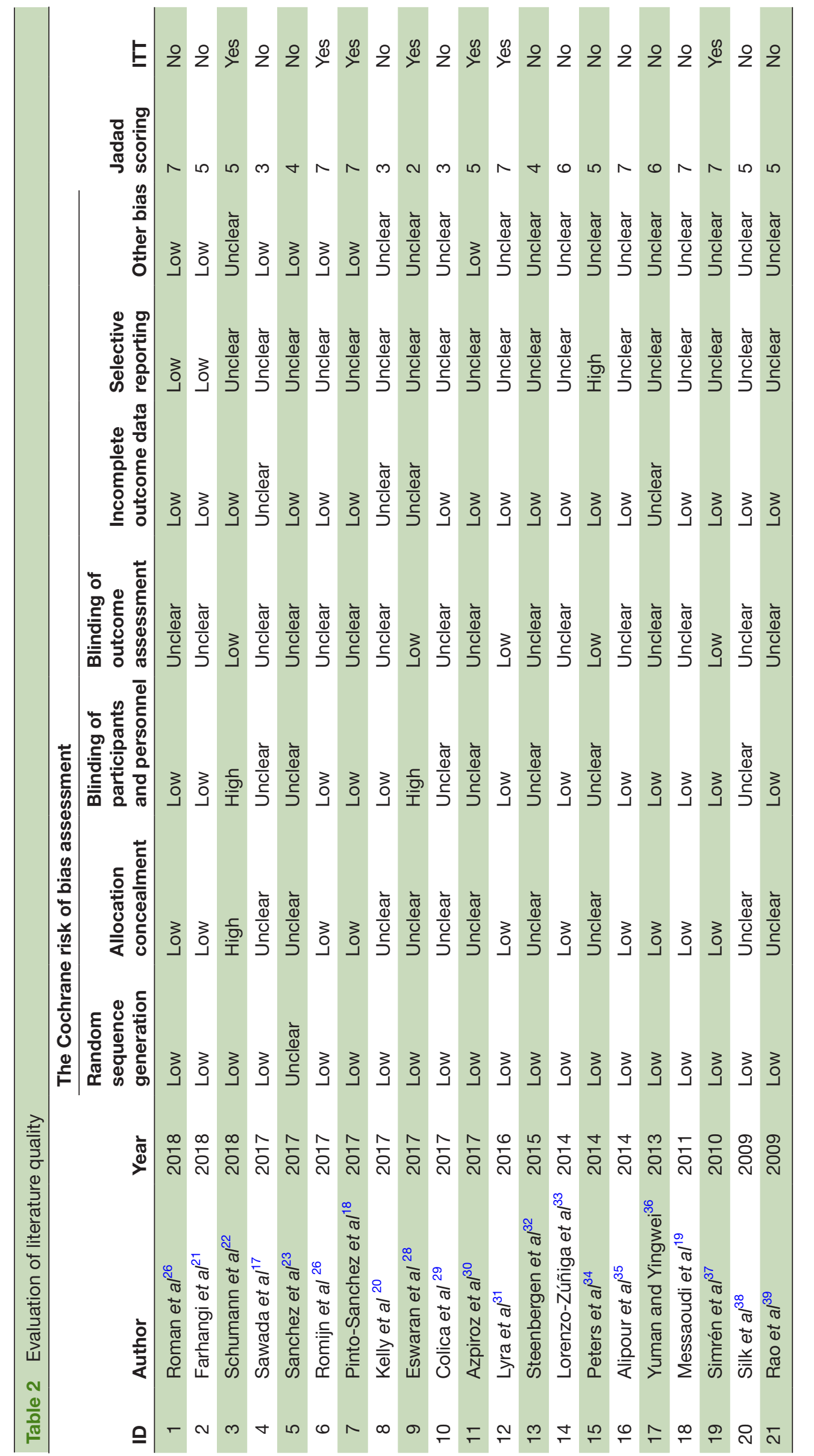

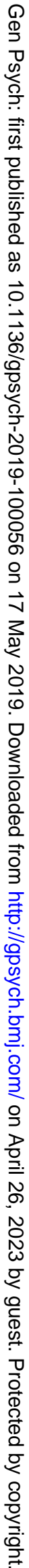


Table 3 Adverse effects and reasons for withdrawal and/or dropout

\begin{tabular}{|c|c|c|c|c|c|}
\hline \multirow[b]{2}{*}{ ID } & \multirow[b]{2}{*}{ Author } & \multirow[b]{2}{*}{ Year } & \multirow[b]{2}{*}{ Adverse events (n) } & Reasons for withdrawal & \multirow[b]{2}{*}{ Control group (n ) } \\
\hline & & & & Intervention group (n) & \\
\hline 1 & Roman et $a l^{26}$ & 2018 & Intestinal discomfort (2) & $\begin{array}{l}\text { Reasons unrelated to the } \\
\text { intervention (2) }\end{array}$ & Non-therapeutic adherence (2) \\
\hline 2 & Farhangi et al ${ }^{21}$ & 2018 & No serious adverse events & $\begin{array}{l}\text { Did not consume the supplement } \\
\text { (1) }\end{array}$ & $\begin{array}{l}\text { Received anti-inflammatory } \\
\text { medication (2), diet change } \\
\text { (1), did not consume the } \\
\text { supplement (3) }\end{array}$ \\
\hline & & & $\begin{array}{l}\text { Yoga group: a newly diagnosed } \\
\text { deep leg vein thrombosis (1), } \\
\text { back pain (1) }\end{array}$ & & \\
\hline 5 & Sanchez et $a l^{23}$ & 2017 & No data provided & $\begin{array}{l}\text { Poor compliance to the treatment } \\
\text { (1) }\end{array}$ & \\
\hline 6 & Romijn et $a l^{27}$ & 2017 & Dry mouth, sleep disruption & $\begin{array}{l}\text { Antibiotic use (1), antidepressant } \\
\text { use (2), participant choice (4) }\end{array}$ & $\begin{array}{l}\text { Antibiotic use (2), stressful life } \\
\text { events (1) }\end{array}$ \\
\hline 7 & $\begin{array}{l}\text { Pinto-Sanchez } \\
\text { et } a l^{18}\end{array}$ & 2017 & $\begin{array}{l}\text { No serious adverse events } \\
\text { related to study product }\end{array}$ & $\begin{array}{l}\text { Antibiotic use (3), antidepressant } \\
\text { use (1) }\end{array}$ & $\begin{array}{l}\text { Antibiotic use (1), } \\
\text { antidepressant use (1) }\end{array}$ \\
\hline 8 & Kelly et $\left.a\right|^{20}$ & 2017 & Side effects were negligible & No data provided & \\
\hline 9 & Eswaran et $a l^{28}$ & 2017 & No data provided & $\begin{array}{l}\text { Lost to follow-up: not returning } \\
\text { calls (1), started antibiotics (1), } \\
\text { too expensive (1); discontinued } \\
\text { intervention: too limiting (1), } \\
\text { moved out of state (1) }\end{array}$ & $\begin{array}{l}\text { Discontinued intervention: } \\
\text { failed to make symptom } \\
\text { reports ( } 2 \text { ) }\end{array}$ \\
\hline 12 & Lyra et $a l^{31}$ & 2016 & $\begin{array}{l}\text { Treatment-emergent AEs: GI } \\
\text { disorders, gastroenteritis and } \\
\text { influenza } \\
\text { IP-related AEs: mild GI } \\
\text { symptoms ( } 7 \text { placebo, } 7 \text { low } \\
\text { dose and } 9 \text { high dose) } \\
\text { Two SAE cases: pneumonia and } \\
\text { syncope; neither was associated } \\
\text { with the IP or any trial procedure }\end{array}$ & $\begin{array}{l}\text { Low dose-adverse event (4), } \\
\text { lost to follow-up (3), other (4), } \\
\text { protocol violation (1), } \\
\text { withdrawal of consent (5) } \\
\text { High dose-adverse event (10), } \\
\text { other (3), } \\
\text { protocol violation (1), withdrawal } \\
\text { of consent (4) }\end{array}$ & $\begin{array}{l}\text { Adverse event (3), } \\
\text { lost to follow-up (2), } \\
\text { other (2), } \\
\text { protocol violation (3), } \\
\text { withdrawal of consent (6) }\end{array}$ \\
\hline 13 & $\begin{array}{l}\text { Steenbergen et } \\
\left.\mathrm{a}\right|^{32}\end{array}$ & 2015 & No data provided & No data provided & \\
\hline 14 & $\begin{array}{l}\text { Lorenzo-Zúñiga } \\
\text { et } a l^{33}\end{array}$ & 2014 & No adverse drug reactions & $\begin{array}{l}\text { High dose: loss to follow-up (3), } \\
\text { discontinued intervention ( } 3 \text { ) } \\
\text { Low dose: loss to follow-up (3), } \\
\text { discontinued intervention (3) }\end{array}$ & $\begin{array}{l}\text { Loss to follow-up (5), } \\
\text { discontinued intervention (5) }\end{array}$ \\
\hline 15 & Peters et $a^{34}$ & 2014 & No data provided $\ddagger$ & No data provided & \\
\hline 16 & Alipour et $a l^{35}$ & 2014 & No adverse effects & Did not follow the study protocol (8 & $8 / 6)$ \\
\hline 17 & $\begin{array}{l}\text { Yuman and } \\
\text { Yingwei }^{36}\end{array}$ & 2013 & No data provided & No data provided & \\
\hline
\end{tabular}


Table 3 Continued

\begin{tabular}{|c|c|c|c|c|c|}
\hline \multirow[b]{2}{*}{ ID } & \multirow[b]{2}{*}{ Author } & \multirow[b]{2}{*}{ Year } & \multirow[b]{2}{*}{ Adverse events (n) } & \multirow{2}{*}{$\begin{array}{l}\text { Reasons for withdrawal } \\
\text { Intervention group (n) }\end{array}$} & \multirow[b]{2}{*}{ Control group (n ) } \\
\hline & & & & & \\
\hline 18 & Messaoudi et al ${ }^{19}$ & 2011 & No data provided & No data provided & \\
\hline 20 & Silk et $a l^{\beta 8}$ & 2009 & $\begin{array}{l}\text { Moderate diarrhoea (1-3.5 g } \\
\text { placebo), mild nausea (1-7.0 g } \\
\text { placebo, } 1-3.5 \mathrm{~g} \text { prebiotic) }\end{array}$ & $\begin{array}{l}\text { Felt the study was too } d \\
\text { diarrhoea (1), took part ir } \\
\text { commercially available } p\end{array}$ & $\begin{array}{l}\text { (3), felt the placebo caused } \\
\text { probiotic study (1), took a } \\
\text { reparation during baseline (2) }\end{array}$ \\
\hline 21 & Rao et $a l^{39}$ & 2009 & No significant adverse events & Reasons unrelated to the & tion (4) \\
\hline
\end{tabular}

GI disorders refer to gastrointestinal tract disorders, including abdominal discomfort, abdominal distension, abdominal pain, constipation, diarrhoea and flatulence.

${ }^{*}$ None of these events were adjudged to relate to the study interventions. †Did not provide details.

$\ddagger$ One patient ceased the whey challenge (treatment first received) prematurely because of intolerable symptoms after lunch on day 2 . $\mathrm{AE}$, adverse effect; FODMAP, fermentable oligosaccharides, disaccharides, and monosaccharides and polyols; GI, gastrointestinal ; IP, investigational product; SAE, serious adverse event; scFOS, short-chain fructooligosaccharides.

improve the anxiety symptoms by regulating the intestinal flora through non-probiotic methods.

\section{Adverse effects and dropouts}

Most of the studies did not report serious adverse events, and only four studies reported mild adverse effects such as dry mouth, senestopathia and diarrhoea. Schumann and colleagues ${ }^{22}$ reported two serious events related to major depressive episodes and deep leg vein thrombosis, but none of these events were determined to relate to the study interventions. In short, no matter which intervention was taken, the probability of serious adverse reactions was extremely low, and it is safe to improve the anxiety symptoms by regulating the intestinal flora. Besides, the dropout rates did have significant impacts on the results. The details of side effects and reasons for dropout are shown in table 3 .

\section{DISCUSSION}

\section{Main findings}

First of all, more than half of the 21 studies included in this paper showed that regulating intestinal flora can effectively improve anxiety symptoms. Of the 14 studies that used probiotics as the intervention, $36 \%$ of the studies were effective, while six of the seven studies

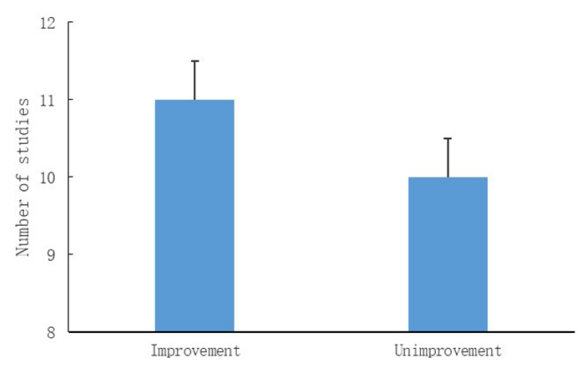

Figure 2 Outcomes of included studies. 11 of 21 studies showed that regulation of intestinal microbiota could improve anxiety symptoms, while 10 studies didn't show positive effects. using non-probiotics as interventions were effective, and the effective rate was $86 \%$. As for the five studies that used the TAU plus IRIF as interventions, only studies that conducted non-probiotic ways were positive; and non-probiotic interventions were also more effective in the studies that used IRIF alone, for $80 \%$ of studies could improve anxiety symptoms in the studies that performed non-probiotic interventions while $45 \%$ were effective in the studies that used probiotic ways. So we can easily find that although we can regulate the intestinal flora in two ways, the non-probiotic intervention is significantly better than the probiotic intervention. The reasons for this result may be as follows: (A) The energy source of gut microbiota growth is mainly food. ${ }^{24}$ Adjusting the gut microbiota through modulating dietary structure can directly change the energy supplying structure of gut microbiota and this plays a decisive role in the growth of gut microbiota, so the effect is obvious. (B) Although the studies all conducted probiotic interventions, the species of the probiotics were diverse and there were survival competitions in implanted flora and primitive flora, which may lead to not all the imported probiotics being effectively implanted. (C) Most intervention times of included studies were $4-8$ weeks. This might be too short to significantly increase the abundance of the imported microbiota, so that the subjects' original intestinal flora could not be effectively adjusted.

Second, $67 \%$ of the studies used probiotic intervention to regulate intestinal flora, while only $33 \%$ of the studies used non-probiotic ways such as low FODMAPS, scFOS and supplementary resistance dextrin. On the one hand, this indicates that more and more researchers have realised that microflora plays an increasingly important role in human health, but on the other hand, the function of diets in daily life has been neglected by people. As mentioned above, the effect of dietary structure adjustment is better than that of probiotic supplements. In the future, more attention can be paid to the regulation of intestinal flora through non-probiotic ways, or 
the combination of probiotic and non-probiotic means, which may have unexpected effects.

In addition, the subjects were patients with chronic diseases comorbid with anxiety symptoms or healthy individuals. Chronic diseases included IBS, CFS, RA, obesity and fibromyalgia. Sixty-seven per cent of chronic disease subjects were patients with IBS, indicating that anxiety symptoms are common in intestinal-related chronic diseases. After reviewing the studies about the pathological mechanism of IBS published in recent years, Raskov and colleagues ${ }^{25}$ found that the gut-brain axis played a central role in the persistence of IBS and the microbiota played a key role. In the study, the improvement of anxiety symptoms in patients with IBS by adjusting intestinal flora was further evidence of the gut-brain axis mechanism. Last but not least, the vast majority of studies have not reported serious adverse events related to interventions, regardless of what kinds of interventions were conducted. Another point that should be paid attention to was that $67 \%$ of six articles about healthy individuals have shown positive effects on anxiety symptoms, this may be strong evidence to support the hypothesis that anxiety symptoms can be relieved by modulating gut microbiota.

Only four studies reported mild adverse reactions such as dry mouth, internal perceptual discomfort and diarrhoea. In summary, more than half of the studies have shown that the intestinal flora could be modulated to alleviate anxiety symptoms and was extremely safe.

\section{Limitations}

Due to the differences in the research design types, subjects, interventions and anxiety assessment scales of the 21 articles included, the overall heterogeneity was too large and it was not suitable for meta-analysis. Fifty per cent of the 10 studies on IBS showed that the interventions were effective. Therefore, for patients with IBS, more studies are needed to verify whether it is possible to clinically treat the anxiety symptoms of patients with IBS by regulating intestinal flora. We did not register on PROSPERO whether the individuals had different kinds of diseases or were healthy individuals, rather we recorded all as having the same symptom-anxiety.

\section{Implications}

In the clinical treatment of anxiety symptoms, in addition to the use of psychiatric drugs for treatment, we can also consider regulating intestinal flora to alleviate anxiety symptoms. Especially for patients with somatic diseases who are not suitable for the application of psychiatric drugs for anxiety treatment, probiotic methods and/or non-probiotic ways like low FODMAPs can be applied flexibly according to clinical conditions. However, there are still some studies showing that the effect of regulating intestinal flora to improve anxiety symptoms is limited. Therefore, more relevant clinical intervention studies should be carried out with the unified anxiety assessment scales and statistical methods being used to clarify the relationship between intestinal flora adjustment and improvement of anxiety symptoms.

Correction notice This article has been corrected since it was first published. This article was not published under an Open Access licence. This has now been corrected.

Acknowledgements The authors thank the translators and reviewers of this article.

Contributors BY: retrieval and screening of literature, bias assessment, data entry, statistical analysis and writing the paper. JW: retrieval and screening of literature, and bias assessment. PJ: bias assessment and guidance of statistical analysis. JC: thesis topic selection and writing modification.

Funding National Natural Science Foundation of China $(81571326,81501153)$ and National Key R\&D Program of China (2017YFC0909200).

Competing interests None declared.

Patient consent for publication Not required.

Provenance and peer review Not commissioned; externally peer reviewed.

Data sharing statement № additional data are available.

Open access This is an open access article distributed in accordance with the Creative Commons Attribution Non Commercial (CC BY-NC 4.0) license, which permits others to distribute, remix, adapt, build upon this work non-commercially, and license their derivative works on different terms, provided the original work is properly cited, appropriate credit is given, any changes made indicated, and the use is non-commercial. See: http://creativecommons.org/licenses/by-nc/4.0/.

\section{REFERENCES}

1. Remes O, Brayne C, van der Linde R, et al. A systematic review of reviews on the prevalence of anxiety disorders in adult populations. Brain Behav 2016;6:e00497.

2. Bandelow B, Michaelis S. Epidemiology of anxiety disorders in the 21st century. Dialogues Clin Neurosci 2015;17:327-35.

3. Neuropsychological and Behavioral Neurology Group of the Chinese Medical Association Neurology branch. Expert consensus on diagnosis and treatment of anxiety, depression and somatization symptoms in general hospitals. Chinese Journal of Neurology 2016;49:908-17.

4. Carding S, Verbeke K, Vipond DT, et al. Dysbiosis of the gut microbiota in disease. Microbial Ecology in Health \& Disease $2015 ; 26$.

5. Lemaitre B, Nicolas E, Michaut L, et al. The dorsoventral regulatory gene cassette spätzle/Toll/cactus controls the potent antifungal response in Drosophila adults. Cell 1996;86:973-83.

6. Takeda K, Akira S. TLR signaling pathways. Seminars in Immunology 2004;16:3-9.

7. Zhu C, Xu J, Lin Y, et al. Loss of microglia and impaired BrainNeurotrophic factor signaling pathway in a comorbid model of chronic pain and depression. Front Psychiatry 2018;9.

8. Sudo N, Chida Y, Aiba Y, et al. Postnatal microbial colonization programs the hypothalamic-pituitary-adrenal system for stress response in mice. J Physiol 2004;558:263-75.

9. Mohajeri MH, La Fata G, Steinert RE, et al. Relationship between the gut microbiome and brain function. Nutr Rev 2018;76:481-96.

10. Foster JA, McVey Neufeld K-A. Gut-brain axis: how the microbiome influences anxiety and depression. Trends Neurosci 2013;36:305-12.

11. Chen J. Comorbidity investigations of gastrointestinal disorders with mental disorders in patients or animals. J Depress Anxiety 2017;06.

12. Neufeld KM, Kang N, Bienenstock J, et al. Reduced anxiety-like behavior and central neurochemical change in germ-free mice. Neurogastroenterol Motil 2011;23:255-e119.

13. Clarke G, Grenham S, Scully P, et al. The microbiome-gut-brain axis during early life regulates the hippocampal serotonergic system in a sex-dependent manner. Mol Psychiatry 2013;18:666-73.

14. Desbonnet L, Clarke G, Shanahan F, et al. Microbiota is essential for social development in the mouse. Mol Psychiatry 2014;19:146-8.

15. Arentsen $\mathrm{T}$, Raith $\mathrm{H}$, Qian $\mathrm{Y}$, et al. Host microbiota modulates development of social preference in mice. Microb Ecol Health Dis 2015;26.

16. Collins SM, Kassam Z, Bercik P. The adoptive transfer of behavioral phenotype via the intestinal microbiota: experimental evidence and clinical implications. Curr Opin Microbiol 2013;16:240-5. 
17. Sawada D, Kawai T, Nishida K, et al. Daily intake of Lactobacillus gasseri CP2305 improves mental, physical, and sleep quality among Japanese medical students enrolled in a cadaver dissection course. $J$ Funct Foods 2017:31:188-97.

18. Pinto-Sanchez MI, Hall GB, Ghajar K, et al. Probiotic Bifidobacterium longum NCC3001 reduces depression scores and alters brain activity: a pilot study in patients with irritable bowel syndrome. Gastroenterology 2017;153:448-59.

19. Messaoudi M, Lalonde R, Violle N, et al. Assessment of psychotropic-like properties of a probiotic formulation (Lactobacillus helveticus R0052 and Bifidobacterium longum R0175) in rats and human subjects. Br J Nutr 2011;105:755-64.

20. Kelly JR, Allen AP, Temko A, et al. Lost in translation? The potential psychobiotic Lactobacillus rhamnosus (JB-1) fails to modulate stress or cognitive performance in healthy male subjects. Brain Behav Immun 2017;61:50-9.

21. Farhangi MA, Javid AZ, Sarmadi B, et al. A randomized controlled trial on the efficacy of resistant dextrin, as functional food, in women with type 2 diabetes: targeting the hypothalamic-pituitary-adrenal axis and immune system. Clin Nutr 2018;37:1216-23.

22. Schumann D, Langhorst J, Dobos G, et al. Randomised clinical trial: yoga vs a low-FODMAP diet in patients with irritable bowel syndrome. Aliment Pharmacol Ther 2018;47:203-11.

23. Sanchez M, Darimont C, Panahi S, et al. Effects of a diet-based weight-reducing program with probiotic supplementation on satiety efficiency, eating behaviour traits, and psychosocial behaviours in obese individuals. Nutrients 2017;9.

24. Milani C, Duranti S, Bottacini F, et al. The first microbial colonizers of the human gut: composition, activities, and health implications of the infant gut microbiota. Microbiol Mol Biol Rev 2017;81:e00036-17.

25. Raskov H, Burcharth J, Pommergaard $\mathrm{H}-\mathrm{C}$, et al. Irritable bowel syndrome, the microbiota and the gut-brain axis. Gut Microbes 2016;7:365-83.

26. Roman $\mathrm{P}$, Estévez AF, Miras A, et al. A pilot randomized controlled trial to explore cognitive and emotional effects of probiotics in fibromyalgia. Scientific Reports 2018;8.

27. Romijn AR, Rucklidge JJ, Kuijer RG, et al. A double-blind, randomized, placebo-controlled trial of Lactobacillus helveticus and Bifidobacterium longum for the symptoms of depression. Aust N Z J Psychiatry 2017;51:810-21.

28. Eswaran S, Chey WD, Jackson K, et al. A Diet Low in Fermentable Oligo-, Di-, and Monosaccharides and Polyols Improves Quality of
Life and Reduces Activity Impairment in Patients With Irritable Bowel Syndrome and Diarrhea. Clin Gastroenterol Hepatol 2017;15:1890-9.

29. Colica C, Avolio E, Bollero P, et al. Evidences of a new Psychobiotic formulation on body composition and anxiety. Mediators Inflamm 2017;2017:1-10.

30. Azpiroz F, Dubray C, Bernalier-Donadille A, et al. Effects of scFOS on the composition of fecal microbiota and anxiety in patients with irritable bowel syndrome: a randomized, double blind, placebo controlled study. Neurogastroenterol Motil 2017;29.

31. Lyra A, Hillilä M, Huttunen T, et al. Irritable bowel syndrome symptom severity improves equally with probiotic and placebo. World $\mathrm{J}$ Gastroenterol 2016;22:10631-42.

32. Steenbergen L, Sellaro R, van Hemert S, et al. A randomized controlled trial to test the effect of multispecies probiotics on cognitive reactivity to sad mood. Brain Behav Immun 2015;48:258-64

33. Lorenzo-Zúñiga $\mathrm{V}$, Llop $\mathrm{E}$, Suárez $\mathrm{C}$, et al. I.31, a new combination of probiotics, improves irritable bowel syndrome-related quality of life. World J Gastroenterol 2014;20:8709-16.

34. Peters SL, Biesiekierski JR, Yelland GW, et al. Randomised clinical trial: gluten may cause depression in subjects with non-coeliac gluten sensitivity - an exploratory clinical study. Aliment Pharmacol Ther 2014;39:1104-12.

35. Alipour B, Homayouni-Rad A, Vaghef-Mehrabany E, et al. Effects of Lactobacillus casei supplementation on disease activity and inflammatory cytokines in rheumatoid arthritis patients: a randomized double-blind clinical trial. Int J Rheum Dis 2014;17:519-27.

36. Yuman Y, Yingwei Z. Study on intestinal flora changes and nutritional intervention in nursing students before examination in higher vocational colleges. Chinese Journal of Health Psychology 2013;21:1904-5.

37. Simrén M, Ohman L, Olsson J, et al. Clinical trial: the effects of a fermented milk containing three probiotic bacteria in patients with irritable bowel syndrome - a randomized, double-blind, controlled study. Aliment Pharmacol Ther 2010;31:218-27.

38. Silk DBA, Davis A, Vulevic J, et al. Clinical trial: the effects of a trans-galactooligosaccharide prebiotic on faecal microbiota and symptoms in irritable bowel syndrome. Aliment Pharmacol Ther 2009;29:508-18.

39. Rao AV, Bested AC, Beaulne TM, et al. A randomized, double-blind, placebo-controlled pilot study of a probiotic in emotional symptoms of chronic fatigue syndrome. Gut Pathog 2009;1.

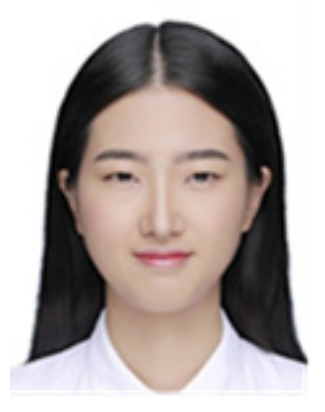

Beibei Yang is currently studying on a master's program in Shanghai Mental Health Center affiliated to Shanghai Jiao Tong University School of Medicine. Her interests include the effects of early stress on neuropsychiatric systems and the pathophysiological mechanisms of brain-gut axis in mental diseases. 